\title{
THE ORDER-DUAL OF A TRL GROUP, I
}

\author{
J. B. MILLER
}

(Received 13 October 1976)

Communicated by E. Strzelecki

\begin{abstract}
Conditions are found for several intrinsically defined partial orders on $\mathfrak{E b}$, the vector space of order-bounded additive functionals on a commutative pogroup, to have Riesz interpolation properties, and to make $\mathfrak{E b}$ a TRL group.

Subject classification (Amer. Math. Soc. (MOS) 1970): primary 06 A 60, 46 A 40; secondary54 F 05, 06 A 75.
\end{abstract}

\section{Introduction}

We begin a study of the vector lattice $\mathfrak{Q b}$ of all order-bounded additive functionals on a commutative partially ordered group $G$, with particular attention to tight Riesz properties of $\mathfrak{L b}$. $G$ is assumed to be an $l$-group with respect to a partial order $\preccurlyeq$, and to carry a compatible tight Riesz order and its open-interval topology. Thus besides the usual notion of positivity for a functional $f \in \mathfrak{Q b}$ there are others, some of which (here written $\leqslant, \leqslant_{0}, \preccurlyeq_{\mathrm{a}}$ ) we describe.

A fundamental theorem due to $F$. Riesz describes the vector-lattice structure of $\mathfrak{Q b}$ under its principal partial order $\leqslant$. We show that two orders $\leqslant$ and $\leqslant_{\mathrm{o}}$ are determining orders for this $\preccurlyeq$. The main aim of the paper is to formulate conditions under which $\leqslant$ on $\mathfrak{E b}$ is a compatible tight Riesz order for $\preccurlyeq$. The interest in this question stems from the fact that, by Riesz's formula, the lattice operations on $\mathfrak{L b}$ with respect to $\preccurlyeq$ are not pointwise on $G^{+}$; this is unlike the situation in most previously studied examples of compatible tight Riesz orders on $l$-groups. Two types of conditions are found; one based on compactness properties in $G\left(9^{\circ}\right.$ and $\S 5)$, the other on properties of basic elements of $G\left(10^{\circ}\right)$. The latter are the more delicate.

We also find sufficient conditions for $\leqslant$ to be non-secular $\left(9^{\circ}, 11^{\circ}, 12^{\circ}\right)$.

By examples it is shown that not all continuous additive functionals need be order-bounded $\left(4^{\circ}, 5^{\circ}\right)$.

Thanks are due to Robert Redfield who supplied the present form of Theorem $10^{\circ}$ and the example in $13^{\circ}$, thus substantially improving an earlier version of this paper.

(C) Copyright Australian Mathematical Society 1978

Copyright. Apart from any fair dealing for scholarly purposes as permitted under the Copyright Act, no part of this JOURNAL may be reproduced by any process without written permission from the Treasurer of the Australian Mathematical Society 
Functionals $f$ which are positive with respect to $\preccurlyeq$, but not with respect to some compatible tight Riesz order for $\preccurlyeq$, lie on the surface of the positive cone of $\mathfrak{L b}$, so a study of such orders gives information about surface structure. A subsequent paper will deal with these questions.

\section{Preliminaries}

2.1 We summarize some definitions and results which are needed later. All order symbols $<,<_{0}, \prec, \ldots$ in this paper should be read as excluding equality, with $\leqslant$ meaning " $<$ or $=$ ", and so on. The $(m, n)$ tight Riesz property for a poset $(X, \leqslant)$, abbreviated TR $(m, n)$, asserts the following: For any set of elements $a_{i}, b_{j}$ $(i=1,2, \ldots, m ; j=1,2, \ldots, n)$ in $X$ such that $a_{i}<b_{j}$ for all $i, j$, there exists $x \in X$ such that $a_{i}<x<b_{j}$ for all $i, j$. We have

$$
\operatorname{TR}(2,2) \Rightarrow \operatorname{TR}(1,2) ; \operatorname{TR}(1,2) \Rightarrow \operatorname{TR}(1,1) ; \operatorname{TR}(1,2) \Leftrightarrow \operatorname{TR}(2,1)
$$

when $X$ is a pogroup; TR $(1,2)$ does not imply TR $(2,2)$. The loose Riesz property $\operatorname{LR}(m, n)$ is defined by replacing $<$ by $\leqslant$ at each occurrence. For any order $\leqslant$ on $X$, its associated preorder $\preccurlyeq$ is defined thus:

$$
x \preccurlyeq y \text { if and only if }(\forall u \in X)[u<x \Rightarrow u<y] \&(\forall t \in X)[t>y \Rightarrow t>x] .
$$

When $(X, \leqslant)$ is a pogroup, this is equivalent to saying:

$$
z \geqslant 0 \text { if and only if } a>0 \Rightarrow a+z>0 \text {; }
$$

that is, the positive wedge of $\leqslant$ is got by adjoining to the positive cone of $\leqslant$ all the pseudopositives of $\leqslant$. We consider only cases where $\preccurlyeq$ is a partial order. We call $\leqslant$ a determining order for $\preccurlyeq$. A partial order may have many determining orders.

A tight Riesz group (abbreviated TR group) is here defined to be a directed commutative $\nmid$ pogroup $(G, \leqslant)$ with the TR $(1,2)$ property, and without pseudozeros, so that $(G, \preccurlyeq)$ is likewise a directed pogroup. We call $\leqslant$ a compatible tight Riesz order (CTRO) for $\preccurlyeq$. It is generally assumed that $G \neq(0)$ and neither $\leqslant$ nor $\preccurlyeq$ is trivial. The open-interval topology $\mathscr{U}$ defined from $\leqslant$ makes $(G, \mathscr{U})$ a non-discrete Hausdorff topological group, non-compact though quite possibly locally compact. Thus a TR group has a structure $(G, \leqslant, \preccurlyeq, \mathscr{U})$. By a $T R(2,2)$ group we mean a TR group for which $\leqslant$ is $\operatorname{TR}(2,2)$. For elementary consequences of these various definitions see Loy and Miller (1972) or Cameron and Miller (1975). We write $P=\{x \in G: x \geqslant 0\}, P^{*}=P \backslash\{0\}, G^{+}=\{x: x \geqslant 0\}$ for the positive cones. Orderintervals are written $(a, b)=\{x: a<x<b\},[a, b]=\{x: a \leqslant x \leqslant b\}$, and similarly $((a, b)), \llbracket a, b \rrbracket$ for $\preccurlyeq$. The intervals $(a, b), a<b$, form a base for $\mathscr{U}$.

By a $T R L$ group we mean a structure $(G, \leqslant, \preccurlyeq, \mathscr{U})$ in which $(G, \leqslant)$ is a TR group and $(G, \preccurlyeq)$ is an $l$-group, $\preccurlyeq$ being of course the associated order of $\leqslant$ and $\mathscr{U}$

† We assume that all groups in this paper are commutative: "group" means "abelian group". 
being the open-interval topology of $\leqslant$. (By an "l-group", we mean a "latticeordered group" in the usual sense, as in Birkhoff (1967). The lattice operations of $(G, \preccurlyeq)$ are written $\wedge, \vee$.) For a TRL group $G, \leqslant$ is isolated and $(G, \mathscr{U})$ has no compact subgroups other than (0); see Loy and Miller (1972). If $G$ is a TR group for which $\preccurlyeq$ is $\operatorname{LR}(2,2)$ (in particular, if $G$ is a TRL group), then $\leqslant$ is necessarily TR $(2,2)$. See Cameron and Miller (1975).

A TRL group $G$ is called secular (or androgynous) if any of the following pairwise equivalent properties hold:

(i) $G$ contains a pair of elements $x, y$ satisfying

$$
x>0, \quad y \succ 0, \quad x \wedge y=0 .
$$

(ii) The set $\mathrm{\Upsilon}=\left\{x \in G: x^{+}>0, x^{-}>0\right\}$ is non-empty. (Here $x^{+}=x \vee 0$, $x^{-}=-(x \wedge 0)$.)

(iii) $P^{*} \nsubseteq \mathfrak{w}$. (Here $\mathfrak{w}=\{w \succ 0: w \wedge x=0 \Rightarrow x=0\}$ is the set of weak units of $(G, \preccurlyeq)$; we may have $\mathfrak{w}=\varnothing$.)

There are other characterizations; see Miller (1976). The property expresses a certain relationship of $\leqslant$ to its associated order, resulting in $P^{*}$ occupying a greater portion of $G^{+}$than is sometimes desirable; it can lead to computational difficulties. Since $\leqslant$ determines $\preccurlyeq$, it is allowable to call $\leqslant$ secular, rather than $G$. Secular groups are discussed in some detail in Miller (1976).

2.2 For any pogroup $(G, \leqslant)$, its order-dual is the real vector space $\mathfrak{Q b}(G)$ (briefly, $\mathrm{Sb})$ of all order-bounded additive functionals in $G$, that is, additive functions mapping order-intervals of $G$ to bounded subsets of $\mathbf{R}$. When $G$ is a TR group, it does not matter which of its two orders is used here: they produce the same set $\mathfrak{L b}$. However, when it comes to ordering $\mathfrak{L} \mathfrak{b}$, as usual by ordering functionals pointwise on the positive cone of $G$, several possibilities arise. For $f \in \mathfrak{Q b}$ we shall write

$$
\begin{array}{lll}
f>0 & \text { if and only if } & (\forall x \in G)[x>0 \Rightarrow f(x)>0], \\
f>{ }_{0} 0 & \text { if and only if } & (\forall x \in G)[x>0 \Rightarrow f(x)>0], \\
f \geqslant 0 & \text { if and only if } & (\forall x \in G)[x>0 \Rightarrow f(x) \geqslant 0], \\
f \geqslant_{\mathrm{a}} 0 & \text { if and only if } & (\forall x \in G)[x>0 \Rightarrow f(x) \geqslant 0] ;
\end{array}
$$

and $f \leqslant g$ will mean $g-f>0$ or $g=f$, etc. These definitions make $\mathfrak{L b}$ a partially ordered vector space with respect to each of $\leqslant, \leqslant_{0}, \preccurlyeq, \preccurlyeq_{a}$.

When $G$ is a TRL group it is natural to think of the $l$-group structure of $(G, \preccurlyeq)$ as the dominating one, since much is known about $l$-groups. If we accept this view then $\preccurlyeq$ in (4) is the natural order to place on $\mathscr{S b}$. Notice that the orders $\leqslant$ and $\preccurlyeq$ in (2) and (4) are wholly determined by $\preccurlyeq$ on $G$, that is, are defined for any pogroup $(G, \preccurlyeq)$ whether or not $\preccurlyeq$ is an associated order. Our principal concern is with (2) and (4); nevertheless, the $\leqslant$-structure on $G$ is relevant. 
We write $\mathfrak{L}^{+}$for the positive cone of $\preccurlyeq$ in $\mathfrak{E b}$. It is clear that

$$
f>0 \Rightarrow f>{ }_{0} 0 \Rightarrow f \succ_{\mathrm{a}} 0 \text { and } f>0 \Rightarrow f \succ 0 \Rightarrow f \succ_{\mathrm{a}} 0 .
$$

From results due to Hayes $(1962)$ we know that $\operatorname{Hom}(G, \mathbf{R})$ contains nonzero elements, for any non-trivial group $G$. Bonsall (1954) has pointed out that if $(G, \preccurlyeq)$ is an everywhere non-archimedean pogroup, that is, if

$$
G=\{x: \text { there exists } a \geqslant 0 \text { such that }-a \preccurlyeq n x \preccurlyeq a \text { for all } n \in \mathbf{N}\},
$$

then $\mathfrak{L}^{+}=(0)$. On the other hand, another result of Hayes (1962) shows that $\mathfrak{Q}^{+}$ contains non-zero elements if $G$ has a strong unit. When $G$ is a locally compact TR group with $\leqslant$ isolated, Mackey's theorem shows that the continuous additive functionals on $G$ are sufficiently numerous to separate points. (Compare Hewitt and Ross (1963), Theorems (24.34) and (24.35).) It is easy to construct examples in which $\leqslant$ on $\mathfrak{Q b}$ is non-trivial; see $6^{\circ}$ below.

We note some preliminary results for the structure $(G, \leqslant, \preccurlyeq, \mathscr{U})$.

$1^{\circ}$. When $G$ is a $T R$ group, the orders $\preccurlyeq$ and $\preccurlyeq_{\mathrm{a}}$ on $\mathfrak{Q b}$ coincide, and $f \geqslant 0$ implies that $f$ is continuous. We have, for all $f \in \mathfrak{Q b}$,

$$
f>0 \Rightarrow f>_{0} 0 \Rightarrow f>0 \Leftrightarrow f>_{\mathrm{a}} 0 .
$$

Proof. Certainly $f \succcurlyeq_{0}$ implies $f \succcurlyeq_{\mathrm{a}} 0$. We prove that $f \succcurlyeq_{\mathrm{a}} 0$ implies that $f$ is continuous. Let $f \succcurlyeq_{\mathrm{a}} 0$. Suppose $\operatorname{ker}(f)$ meets $P^{*}$, say $f(a)=0$ with $a>0$. Then for any $x \in G, x+(-a, a)$ is a neighbourhood of $x$ on which $f$ is constant. Hence $f$ is continuous. Suppose, on the other hand, that $\operatorname{ker}(f)$ does not meet $P^{*}$, that is $f>{ }_{0} 0$. Let $\left(x_{i}\right)_{i \in I}$ be a net converging to $x$ in $G$. Given any $\varepsilon>0$ in $\mathbf{R}$ choose any $a \in P^{*}$, then $n \in \mathbf{N}$ so that $0<f(a) / n<\varepsilon$, then $b \in G$ such that $0<b<n b<a$, and take $V=(x-b, x+b)$. (The existence of such an element $b$ is easily shown.) Eventually the net is in $V$ and $\left|f\left(x_{i}\right)-f(x)\right|<\varepsilon$; so again $f$ is continuous.

Finally, $f \succcurlyeq_{\mathrm{a}} 0$ implies $f \geqslant 0$. For if $f \geqslant_{\mathrm{a}} 0$ then when $x \geqslant 0$ we can find a net $\left(x_{i}\right)_{i_{\in} I}$ in $P^{*}$ converging to $x$ and continuity of $f$ gives $f(x) \geqslant 0$, so $f \geqslant 0$. The implication (6) is clear. //

$2^{\circ}$. When $(G, \leqslant)$ is a TR group:

(i) If $f \succ 0$ and $f(c) \neq 0$ for some $c>0$, then $f$ does not vanish identically on $(0, c)$.

(ii) If $f>0$ then $\operatorname{ker}(f)$ is a closed convex subgroup of $(G, \preccurlyeq, \mathscr{U})$, and $f(b)>0$ for some $b>0$.

(iii) For $f \in \mathfrak{Q b}, f>{ }_{0} 0$ if and only if $f>0$ and $\operatorname{ker}(f)$ is not open. If $f>{ }_{0} 0$ then $\operatorname{ran}(f)$ is dense in $\mathbf{R}$.

Proof. (i) and (ii) are straightforward. (iii) Clearly $f>_{0} 0$ if and only if $f>0$ and $\operatorname{ker}(f)$ does not meet $P^{*}$. On the other hand, $\operatorname{ker}(f)$ is open if and only if $\operatorname{ker}(f)$ meets $P^{*}$. For if $\operatorname{ker}(f)$ is open then it contains some interval $(a, b)$, and taking 
$a<x<y<b$ we get $y-x \in \operatorname{ker}(f) \cap P^{*}$; conversely, if $\operatorname{ker}(f)$ meets $P^{*}$, say $f(a)=0$, $a>0$, then by (ii) $\operatorname{ker}(f)$ contains the open interval $(a, 2 a)$, hence it has an interior point, hence it is open.

Suppose $f>{ }_{0} 0$; let $0<\varepsilon \in \mathbf{R}$. As in the proof of $1^{\circ}$ there exists $b>0$ with $0<f(b)<\varepsilon$. (For this, $f \succ 0$ does not suffice; we need $f>{ }_{0} 0$.) So if $0<\alpha<\beta$ in $\mathbf{R}$ write $\varepsilon=\frac{1}{2}(\beta-\alpha)$ and find a corresponding $b$. Then $(m-1) f(b) \leqslant \alpha<m f(b)$ for some $m \in \mathbf{N}$, and this implies $\alpha<f(m b)<\beta$. Thus $f\left(P^{*}\right)$ is dense in $\mathbf{R}^{+}$and hence $f(G)$ is dense in $\mathbf{R}$. //

$3^{\circ}$. When $(G, \leqslant)$ is a TR group, each of the orders $\leqslant$ and $\leqslant_{0}$, if non-trivial, is a determining order on $\mathfrak{L b}$ for $\preccurlyeq$, and their cones have bases. The cone $\mathfrak{L}^{+}$of $\preccurlyeq$ has a base if $G$ has a strong unit.

The same conclusions follow for $\leqslant$ and $\preccurlyeq$ on $\mathfrak{Q b}$ if $(G, \preccurlyeq)$ is any l-group.

Proof. Consider $\leqslant_{0}$. By $1^{\circ}, f \leqslant_{0} g$ implies $f \preccurlyeq g$. Now if $h \succ 0$ then

$$
f>{ }_{0} 0 \text { implies } f+h>{ }_{0} 0 \text {, }
$$

for when $x \in P^{*}$ we have $(f+h)(x)=f(x)+h(x)>0 ;(7)$ shows that $h$ is positive in the associated order of $\leqslant 0$. Conversely, suppose $0 \nless h$, so that $h(x)<0$ for some $x>0$. If $f>{ }_{0} 0$ then $f(x) \geqslant 0$ and by multiplying $f$ by a small positive real if necessary we can arrange that $0 \leqslant f(x)<-h(x)$, so $0 \nless f+h$ and hence $f+h \ngtr_{0} 0$. Therefore (7) does not hold. This proves that $\leqslant_{0}$ determines $\preccurlyeq$. The proof for $\leqslant$ is the same.

Concerning bases for the cones, by Peressini (1976), p. 26, it suffices to produce a strictly positive linear functional in each case, that is, a linear map $\alpha: \mathfrak{L b} \rightarrow \mathbf{R}$ such that $f>0 \Rightarrow \alpha(f)>0$, or $f>{ }_{0} 0 \Rightarrow \alpha(f)>0$, or $f>0 \Rightarrow \alpha(f)>0$, respectively. For the first two cases take any $x \in P^{*}$ and define $\alpha(f)=f(x)$. In the third define $\alpha(f)=f(s)$ where $s$ is a strong unit of $G . \dagger$

When $(G, \preccurlyeq)$ is any $l$-group (that is, no determining order for $\preccurlyeq$ is given) the statements about $\leqslant$ and $\preccurlyeq$ on $\mathfrak{Q b}$ still make sense and are proved in the same way. //

For any pogroup $(G, \preccurlyeq)$ (whether or not $\preccurlyeq$ is an associated order) there is F. Riesz's theorem (see, for example, Peressini (1967), §2.3):

If $(G, \preccurlyeq)$ is an $L R(2,2)$ directed pogroup then $(\mathscr{L b}(G), \preccurlyeq)$ is a complete vector lattice, the lattice operations being given (for $a \in G^{+}$) by the formulae

$$
\begin{aligned}
& (f \vee g)(a)=\sup \left\{f(x)+g(y): x, y \in G^{+}, x+y=a\right\}, \\
& (f \wedge g)(a)=\inf \left\{f(x)+g(y): x, y \in G^{+}, x+y=a\right\} ;
\end{aligned}
$$

and

$$
\mathfrak{Q} \mathfrak{b}=\mathfrak{L}^{+}-\mathfrak{l}^{+} .
$$

$\dagger$ The element $s$ is a strong unit for $(G, \leqslant)$ if and only if $\left(\forall x \in G^{+}\right)(\exists n \in N)(x \leqslant n s)$. The order $\leqslant$ and its associated order have the same set of strong units. 
The conditions here are met when $(G, \preccurlyeq)$ is an $l$-group. The conclusions of the theorem are also deducible from the following modified hypothesis: $(G, \leqslant)$ is a TR $(2,2)$ group. The proof is like that for the theorem itself, and uses also $1^{\circ}$ above. Since $\leqslant$ is $\operatorname{TR}(2,2)$ it is also $\operatorname{LR}(2,2)$, and the formulae (8) and (9) also hold in their modified forms

$$
(f \vee g)(a)=\sup \left\{f(x)+g(y): x, y \in P^{*}, x+y=a\right\}
$$

and its dual, $\left(9^{\prime}\right)$.

\section{Examples}

For a TR group $G$ we can form the real vector space $\mathfrak{L} \equiv \mathscr{L}(G)$ of all continuous additive functionals on $G$. By $1^{\circ}$ and (10) we have

$$
\mathfrak{Q b} \subseteq \mathfrak{L} \text {. }
$$

The following two examples illustrate cases where $\mathfrak{L b} \neq \mathfrak{L}$. The first is due to R. H. Redfield.

$4^{\circ}$. Let $G=\mathbf{R} \circ \mathbf{R}$, the lexicographic product of $\mathbf{R}$ with itself, in which $\langle x, y\rangle>0$ if and only if $x>0$ or $x=0, y>0$. Here $\leqslant$ is full and so coincides with its associated order $\preccurlyeq ; G$ is a TRL group. Let $f$ be the $\operatorname{map} f\langle x, y\rangle=y$. Then $f \in \mathfrak{L}$. However, if $A=\{\langle 0, y\rangle: y \in \mathbf{R}\}$ then $A$ is bounded since $\langle-1,0\rangle\langle A\langle\langle 1,0\rangle$, but $f(A)=\mathbf{R}$. Thus $f \notin \mathfrak{Q b}$.

$5^{\circ}$. Let $G$ be the subgroup of $C[0,1]$ consisting of all continuous functions $x$ for which the derivative $x^{\prime}(0)$ exists. Take $\preccurlyeq$ to be the weak pointwise order $(x \geqslant 0$ if and only if $x(t) \geqslant 0$ for all $0 \leqslant t \leqslant 1)$, and define $x>0$ to mean $x(t)>0$ for all $0<t \leqslant 1$. Then $(G, \preccurlyeq)$ is an $l$-subgroup of $(C[0,1], \preccurlyeq)$, though not convex, and

$$
(x \vee y)^{\prime}(0)= \begin{cases}y^{\prime}(0) & \text { (if } x(0)<y(0)), \\ \max \left\{x^{\prime}(0), y^{\prime}(0)\right\} & \text { (if } x(0)=y(0)) .\end{cases}
$$

Moreover, $\leqslant$ is a $\operatorname{TR}(2,2)$ determining order for $\preccurlyeq$. With respect to its openinterval topology $\mathscr{U}$, convergence of a net $\left(x_{i}\right)_{i \in I}$ to $x$ implies that $x_{i}$ converges uniformly to $x$ on $[0,1]$ and $x_{i}(0)=x(0)$ for $i \geqslant$ some $i_{0}$. $G$ is a nonsecular TRL group; $\preccurlyeq$ is archimedean, $\leqslant$ is not eudoxian.

Let $f$ be the map defined by $f(x)=x^{\prime}(0)$; we have $f \in \mathfrak{L} \backslash \mathfrak{L b}$.

Instead, let $G$ be as above, except that $\leqslant$ is now defined thus: $x>0$ means

$$
x(t)>0 \text { if } 0<t<\frac{1}{2}, \quad x(t) \geqslant 0 \text { if } \frac{1}{2} \leqslant t \leqslant 1 \text { or } t=0 .
$$

We still have $f \in \mathfrak{L} \backslash \mathfrak{L} \mathfrak{b}$, but $\leqslant$ is now a secular order for the TRL group $G$. 
$6^{\circ}$. Let $c$ denote the sequence space of all real sequences $\alpha=\left(\alpha_{n}\right)_{n \in N}$ for which the limit

$$
\lambda(\alpha)=\lim _{n \rightarrow \infty} \alpha_{n}
$$

exists; $c$ is a vector lattice, a fortiori a commutative $l$-group, with respect to the weak pointwise order $\preccurlyeq$ on sequences. For any real sequence $\zeta=\left(\zeta_{n}\right)_{n \in \mathbf{N}}$ write

$$
f(\alpha)=\sum_{n=1}^{\infty} \zeta_{n} \alpha_{n}
$$

This equation defines an element $f \in \mathfrak{L}^{+}(c)$ if and only if $\zeta \in l^{1}$ and $\zeta_{n} \geqslant 0$ for all $n$. The general element of $\mathfrak{L}^{+}$has the form $f+\rho \lambda$, where $\rho \in \mathbf{R}^{+}$, so that $\mathfrak{L b}$ can be identified with $l^{1} \oplus(\lambda)$.

For $f$ in (11) we have $f>0$ if and only if $\zeta_{n}>0$ for all $n$; on the other hand, $\lambda \succ 0, \lambda \ngtr 0$. The only lattice homomorphisms in $\mathfrak{L}^{+}$are $\lambda$ and those $f$ for which $\operatorname{supp}(\zeta)$ is a singleton.

Let a filter $\mathscr{F}$ of subsets of $\mathbf{N}$ be given; define $\leqslant$ on $c$ by

$$
\alpha>0 \text { if and only if } \alpha \geqslant 0 \text { and } \operatorname{supp}(\alpha)=\left\{n \in \mathbf{N}: \alpha_{n}>0\right\} \in \mathscr{F} \text {. }
$$

Then $\leqslant$ is a compatible TR $(2,2)$ order for $(c, \preccurlyeq)$. For $f$ in $(11)$ we have

$$
f>{ }_{0} 0 \text { if and only if } \operatorname{supp}(\zeta) \text { meets every set in } \mathscr{F} \text {; }
$$

we have $\lambda x_{0} 0$.

Every sequence $\alpha$ in $c^{+}$for which inf $\alpha_{n}>0$ is a strong unit of $c$; $\mathfrak{Q b}$ has no strong units.

\section{Tight interpolation for $\leqslant$ and $\leqslant$ o}

4.1 The orders $\leqslant$ and $\leqslant_{o}$ on $\mathfrak{L b}$ are TR $(1,1)$, that is, order-dense, since for example if $f<g$ then $f<\frac{1}{2}(f+g)<g$. If $\leqslant$ is non-trivial and TR $(1,2)$ then since its associated order is $\operatorname{LR}(2,2)$, $\leqslant$ is a compatible $\operatorname{TR}(2,2)$ order for $\preccurlyeq$. The same remark applies to $\leqslant_{0}$. Let $\mathscr{T}$ denote the open-interval topology of $\leqslant$ on $\mathfrak{L b}$. We have, in view of previous remarks:

$7^{\circ}$. If $(G, \leqslant, \preccurlyeq, \mathscr{U})$ is a $T R(2,2)$ group (or if $(G, \preccurlyeq)$ is any l-group) and if $\leqslant$ on $\mathfrak{Q b}$ is non-trivial and $T R(1,2)$, then

is a TRL group.

$$
(\mathfrak{Q b}(G), \leqslant, \preccurlyeq, \mathscr{T})
$$

We ask if either order is TR $(1,2)$. First, we note that $\leqslant_{0}$ need not be TR $(1,2)$. This failure is simply illustrated by the following example.

$8^{\circ}$. Take $G=\mathbf{R}^{2}$ with the strong and weak pointwise orders $\leqslant, \preccurlyeq$, and functionals

$$
f\left\langle x_{1}, x_{2}\right\rangle=x_{1}, \quad g\left\langle x_{1}, x_{2}\right\rangle=x_{2} .
$$


We have $f, g \in \mathfrak{L}^{+}$, in fact $f, g>_{0} 0$, and for any $a=\left\langle a_{1}, a_{2}\right\rangle>0$ in $\mathbf{R}^{2}$,

$$
(f \wedge g)(a)=\inf \{f(x)+g(y): 0 \preccurlyeq x, y ; x+y=a\}=0,
$$

the infimum being attained by taking $x=\left\langle 0, a_{2}\right\rangle, y=\left\langle a_{1}, 0\right\rangle$. Thus $f \wedge g=0$, and so $0<{ }_{0} h<_{0} f, g$ is possible for no $h \in \mathfrak{L b}$.

This example also shows that $\wedge$ for $\mathfrak{S b}$ need not be pointwise on $G^{+}$, since $f(a) \wedge g(a)=\min \left\{a_{1}, a_{2}\right\}>0$. If for any TRL group $G$ it is the case that $\wedge$ (and so $\vee$ ) is pointwise on $G^{+}$then $\leqslant$and $\leqslant_{0}$ are $\operatorname{TR}(2,2)$ on $\mathfrak{L b}$. For with $0<f, g$ in $\mathfrak{L b}$ and $a \succ 0$ we should have $0<f(a) \wedge g(a)=(f \wedge g)(a)$, so $f \wedge g>0$, whence

$$
0<\frac{1}{2}(f \wedge g)<f, g
$$

so $\leqslant$ is $\operatorname{TR}(1,2)$, hence $\operatorname{TR}(2,2)$. However, the proviso is rather special, as we have seen. The counterexample shows that $\leqslant_{0}$ is not really the appropriate order to expect to be TR $(2,2)$. For $\leqslant$ the property is more apt, but is still a delicate matter. The remainder of this section deals with the question for $\leqslant$ on $\mathfrak{R b}$. We describe two cases where $\leqslant$, if non-trivial, can be shown to be TR $(2,2)$ : when $(G, \mathscr{U})$ is interval-compact, and when $(G, \preccurlyeq)$ has a basis.

4.2 A TR $(2,2)$ group is called interval-compact if $\llbracket a, b]$ is compact for every $a \preccurlyeq b$. We have

$$
(a, b)^{-}=((a, b))^{-}=\llbracket a, b \rrbracket \text { whenever } a<b
$$

(where - denotes closure); and equivalent formulations of the property are: $(a, b)^{-}$is compact for every $a<b ;(0, a)^{-}$is compact for every $a>0 ; \llbracket 0, a \rrbracket$ is compact for every $a \succcurlyeq 0$.

If $(G, \leqslant, \preccurlyeq, \mathscr{U})$ is an interval-compact $\operatorname{TR}(2,2)$ group then $(G, \preccurlyeq)$ is a latticecomplete $l$-group, and $(G, \mathscr{U})$ is locally compact. For these and related results see Loy and Miller (1972).

$9^{\circ}$ THEOREM. If $G$ is an interval-compact $T R(2,2)$ group then $\leqslant$ on $\mathfrak{Q b}(G)$ if nontrivial is $T R(2,2)$, and $(\mathfrak{Q b}, \leqslant, \preccurlyeq, \mathscr{T})$ is a non-secular TRL group.

Proof. Let $f, g \in \mathfrak{L}^{+}$, and $a \succ 0$ in $G$. From (9) we have

$$
(f \wedge g)(a)=g(a)-\sup \{g(x)-f(x): 0 \preccurlyeq x \preccurlyeq a\} \geqslant 0 .
$$

There exists a net $\left(x_{i}\right)_{i \in I}$ in $\llbracket 0, a \rrbracket$ such that

$$
\lim \left(g\left(x_{i}\right)-f\left(x_{i}\right)\right)=\sup \{g(x)-f(x): 0 \preccurlyeq x \preccurlyeq a\} .
$$

Suppose that $(f \wedge g)(a)=0$. Then $\lim \left(g\left(x_{i}\right)-f\left(x_{i}\right)\right)=g(a)$. Since

$$
g(x)-f(x) \leqslant g(x) \leqslant g(a)
$$

for $x \in \llbracket 0, a \rrbracket$, it follows that

$$
\lim g\left(x_{i}\right)=g(a), \quad \lim f\left(x_{i}\right)=0 .
$$


By assumption, $\llbracket 0, a \rrbracket$ is compact, so replacing $\left(x_{i}\right)_{i \in I}$ by a subset if necessary we can assume that $\lim x_{i}$ exists, $=x_{0} \in\left[0, a \rrbracket\right.$. Since $f$ and $g$ are continuous by $1^{\circ}$, $g(a)=\lim g\left(x_{i}\right)=g\left(x_{0}\right)$ and $0=\lim f\left(x_{i}\right)=f\left(x_{0}\right)$.

Now suppose that $f>0$ and $g>0$. Since $x_{0} \prec a$ would imply $g\left(x_{0}\right)<g(a)$ we have $x_{0}=a$ and hence $0<f(a)=f\left(x_{0}\right)=0$, contradiction. We have thus shown that $f, g>0$ implies $(f \wedge g)(a)>0$ for all $a>0$, that is, $f \wedge g>0$. Therefore $\leqslant$ is TR $(1,2)$, and so $\operatorname{TR}(2,2)$, and $7^{\circ}$ shows that $\mathfrak{E b}$ is a TRL group.

Suppose instead that $f \succ 0$ and $g>0$. The above considerations in this case show that $f(a)=0$ whenever $(f \wedge g)(a)=0$ and $a \succ 0$. Thus $f \succ 0$ and $g>0$ imply $f \wedge g>0$, which means (by 2.1) that $\mathfrak{Q b}$ is non-secular. //

Remark: The trivial order is always TR $(2,2)$; on the other hand, for $\leqslant$ to determine $\preccurlyeq$ on $\mathfrak{Q b}, \leqslant$ must be non-trivial.

A less direct proof of $9^{\circ}$ is possible using $10^{\circ}$ and $11^{\circ}$ below and a result due to Wirth (1975) characterizing interval-compact tight Riesz groups.

4.3 For any $l$-group $(G, \preccurlyeq)$, a basic element is by definition an element $a \in G^{+} \backslash\{0\}$ such that $\llbracket 0, a \rrbracket$ is a fully-ordered subset of $G^{+}$. Alternative characterizations are: (i) The carrier $\tilde{a}$ determined by $a$ is an atom of the carrier lattice C of $G$; (ii) If $0 \preccurlyeq s, t \preccurlyeq a$ and $s \wedge t=0$ then $s=0$ or $t=0$; (iii) $a^{\perp \perp}$ is fully-ordered; (iv) $a^{\Perp}$ is an atom of the lattice $\operatorname{Pol}(G)$ of all polars of $G$. (For any subset $A \subseteq G$, the polar of $A$ is $A^{\perp}=\{x \in G:|x| \wedge|a|=0$ for all $a \in A\}$, and $c^{\perp}=\{c\}^{\perp}$. The polars form a complete Boolean algebra $\operatorname{Pol}(G)$ with respect to inclusion and $\perp$ as complementation. For $c \in G^{+}$the carrier determined by $c$ is $\tilde{c}=\left\{x \in G^{+}: x^{\perp}=c^{\perp}\right\}$. The carriers form a distributive disjunctive lattice $(\mathfrak{C}, \preccurlyeq)$ when ordered by writing $\tilde{a} \preccurlyeq \tilde{b}$ if and only if $a^{\Perp} \subseteq b^{\perp}$.)

A basis of the $l$-group $(G, \preccurlyeq)$ is any subset of $G^{+} \backslash\{0\}$ which is maximal with respect to the property: each element of the subset is basic, and the elements are pairwise disjoint. $G$ has a basis if and only if $\mathfrak{C}$ is atomic, that is, every element $\tilde{x} \in \mathbb{C}$ dominates some atom (it is then the join of the atoms it dominates); equivalently, every $x>0$ dominates some basic element.

The following result is due to R. H. Redfield; it subsumes a number of special cases proved earlier by the author using more complicated arguments.

$10^{\circ}$. TheOREM. Let $(G, \preccurlyeq)$ be any l-group with a basis. Then $\leqslant$ on $\mathfrak{Q b}$, if nontrivial, is $T R(2,2)$, and $\mathfrak{Q b}$ is a TRL group.

Proof. Let $f, g>0$ in $\mathfrak{Q b}$ and suppose $u \succ 0$ in $G$. Then $f \wedge g \geqslant 0$ and we wish to show that $(f \wedge g)(u)>0$. By assumption there exists some basic element $a \preccurlyeq u$. Since $(2 a)^{\sim}=\tilde{a}, 2 a$ is also basic. Take any $x, y \in G^{+}$with $x+y=2 a$; since $\llbracket 0,2 a \rrbracket$ is fully-ordered, either $a \preccurlyeq x$ or $a \preccurlyeq y$ so either $f(x)+g(y) \geqslant f(x) \geqslant f(a)$ or 
$f(x)+g(y) \geqslant g(y) \geqslant g(a)$ and therefore by $(9)$,

$$
(f \wedge g)(2 a) \geqslant \min \{f(a), g(a)\}>0
$$

since $f>0$ and $g>0$. Thus in either case

$$
2(f \wedge g)(u) \geqslant(f \wedge g)(2 a)>0 .
$$

This proves that $f \wedge g>0$ and hence that $\leqslant$ is $\operatorname{TR}(2,2)$. Again $7^{\circ}$ shows that $\mathscr{L b}$ is a TRL group. //

The question of whether $\leqslant$ is non-secular is not so immediately settled in this case as it is in $9^{\circ}$. We have the following sufficient condition.

$11^{\circ}$. Let $(G, \preccurlyeq)$ be any l-group with a basis. Suppose that $\leqslant$ on $\mathfrak{Q b}$ is non-trivial, and that for every $f>0$ in $\mathfrak{Q b}$ there exists a basic element a such that $f(a)>0$. Then $\mathfrak{L b}$ is non-secular.

Proof. We have to show that in $\mathfrak{L b}, f>0$ and $g>0$ imply $f \wedge g>0$. Suppose $a$ is basic and $f(a)>0$. Necessarily $g(a)>0$, and consequently the same argument as in the proof of $10^{\circ}$ leads to $(f \wedge g)(a)>0$. This proves $f \wedge g>0$. //

Call an $l$-group $(G, \preccurlyeq)$ Jaffard projectable if $G$ has a basis, and

$$
G=a^{\perp \perp} \oplus a^{\perp}
$$

for every basic element. Call $(G, \preccurlyeq)$ finitely based if $G$ has a basis, and for every non-zero $x \in G^{+}$there exists no infinite subset of $\{y: 0<y \leqslant x\}$ the elements of which are pairwise disjoint (equivalently: the carrier lattice $\mathbb{C}$ of $G$ is atomic and each non-zero carrier $\tilde{x}$ dominates only finitely many atoms). P. Jaffard (1953) showed that an $l$-group is expressible as a direct sum

$$
\sum_{i \in I} \oplus H_{i}
$$

of fully-ordered convex subgroups if and only if it is Jaffard projectable and finitely based. In this case the $H_{i}$ 's are precisely the principal bipolars $a_{i}^{\perp 1}$, where $\tilde{a}_{i}$ runs through the atoms of $\mathfrak{C}$. Here $I$ need not be finite. From $11^{\circ}$ we deduce:

$12^{\circ}$. Let $(G, \preccurlyeq)$ be a Jaffard projectable and finitely based l-group. Then $\leqslant$, if nontrivial, makes $\mathfrak{Q b}$ a non-secular TRL group.

Proof. If $f$ vanishes on every basic element then by the representation $G=\sum_{i \in I} \oplus a_{i}^{\perp \perp}, f$ vanishes on $G$. Thus $f>0$ implies $f(a)>0$ for some basic element $a$, and $11^{\circ}$ gives the result. //

It is reasonable to conjecture that the condition that $\mathbb{C}$ is finitely based can be dropped from $15^{\circ}$. When $(G, \preccurlyeq)$ is Jaffard projectable its basic subgroup $B$ (the 
subgroup generated by the set of all the basic elements) has $G$ for its lattice-closure (this result is due to R. H. Redfield), so if $g$ vanishes on $B$ it might be supposed that it should vanish on $G$. However, if a continuous functional vanishes on a subset $X$ of a TRL group it need not vanish on $\sup (X)$. In fact the conjecture is false, as the following counterexample (also due to Redfield) shows.

$13^{\circ}$. ExAMPLE of a TRL group $G$ for which $(G, \preccurlyeq)$ is order-dense, Jaffard projectable and archimedean, $G$ is non-secular, but $\leqslant$ on $\mathfrak{Q b}$ is secular. Let $G$ be the subset of $\prod_{k=1}^{\infty} \mathbf{R}$ consisting of functions $x$ of the form

$$
x=s+p,
$$

where $s \in \sum_{k=1}^{\infty} \mathbf{R}$ and $p=\left(p_{k}\right)_{k \in \mathrm{N}}, p_{k}=2^{-k} \alpha_{x}$ for some $\alpha_{x} \in \mathbf{Q}$. Clearly $x$ determines $s$ and $p$ uniquely, and $G$ is an $l$-subgroup of the cardinal product $(\Pi \mathbf{R}, \preccurlyeq)$. Let $\leqslant$ be the strong pointwise order on $G$. Then $(G, \leqslant, \preccurlyeq)$ is a TRL group with the asserted properties. We produce two functionals $f, g \in \mathfrak{Q b}$ such that $f>0, g>0$ and $f \wedge g=0$, namely

$$
f(x)=\alpha_{x}, \quad g(x)=\sum_{k=1}^{\infty} x_{k} .
$$

To prove $f \wedge g=0$, let $a \in G^{+}$. If $a \in \sum \mathbf{R}$ then $f(a)=0$ so $f \wedge g(a)=0$. Suppose $a \notin \sum \mathbf{R}$, and let $\varepsilon>0$ in $\mathbf{R}$; pick $n$ such that $\Sigma_{k>n} 2^{-k} \alpha_{a}<\frac{1}{2} \varepsilon$, define $b \in \Pi \mathbf{R}$ by

$$
b_{k}=\varepsilon / 2^{n} \quad(1 \leqslant k \leqslant n), \quad \alpha_{a} / 2^{k} \quad(k>n),
$$

and let $c=a \vee b-b$. Then $0 \preccurlyeq c \in \sum \mathbf{R}$, so $f(c)=0, g(b)<\varepsilon$ and therefore

Thus $f \wedge g=0$.

$$
0 \leqslant(f \wedge g)(a) \leqslant(f \wedge g)(a \vee b) \leqslant f(c)+g(b)<\varepsilon .
$$

4.4 The question of whether $\leqslant$ on $\mathfrak{L b}$ is $\operatorname{TR}(1,2)$, for $l$-groups $G$ not covered in $9^{\circ}$ and $10^{\circ}$, can be formulated in terms of certain sets of the form

$$
A_{s,}(a)=\{x: 0 \preccurlyeq x \preccurlyeq a \text {, but neither } s \preccurlyeq x \text { nor } x \preccurlyeq t\} .
$$

Here $a$ is some element in $G^{+} \backslash\{0\}$, and $s, t \in((0, a))$. It is found most useful to choose $s, t$ so that $0 \prec s \prec t \prec a$. Let $f>0$ and $g>0$ in $\mathfrak{R b}$, and assume that $(f \wedge g)(a)=0$, and consider the sequence $\left(x_{i}\right)_{i \in I}$ in the proof of $9^{\circ}$, with the properties (14) and (15). If for some $s$ we have $0 \prec s \preccurlyeq x_{i}$ for all $i$ in some cofinal subset $I_{0}$ of $I$, then $0<f(s) \leqslant f\left(x_{i}\right)$ for all $i \in I_{0}$, contradicting (15). (Note that we need $f>0$ here, not merely $f \succ 0$.) Similarly, if $x_{i} \leqslant t \prec a$ for some $t$ and all $i$ in some cofinal subset we get $g\left(x_{i}\right) \leqslant g(t)<g(a)$, contradicting (15). Therefore, for all $s, t \in((0, a)), x_{i}$ is eventually in $A_{s, t}(a)$.

If $A_{s, i}(a) \neq \varnothing$ for all such $s, t$, this means roughly speaking that the net $\left(x_{i}\right)_{i \in I}$ migrates towards the boundary of $\llbracket 0, a \rrbracket$ and away from 0 and $a$ : this can be 
illustrated by considering the group $G=\mathbf{R}^{2}$, taking $a>0$. If, on the contrary, it can be shown that $A_{s, l}(a)=\varnothing$ for some pair $s, t \in((0, a))$ then we have a contradiction implying $(f \wedge g)(a)>0$. Thus

$14^{\circ}$. Let $(G, \preccurlyeq)$ be an l-group. For $\leqslant$ on $\mathfrak{Q b}$ to be $T R(1,2)$ it is sufficient that $G$ satisfy the following condition:

[*] For every $a>0$ in $G$ there exists a pair of elements $s, t \in((0, a))$ such that $A_{s, l}(a)=\varnothing$.

The use of basic elements in $10^{\circ}$ reduces the discussion to the case where $[0, a]$ is fully-ordered; here [*] is satisfied trivially by any $s, t$ such that $0 \prec s \prec t \prec a$. Since the sets $A_{s, l}(a)$ for fixed $a$ do not form a filterbase, [ $\left.{ }^{*}\right]$ does not seem to be a necessary condition.

\section{Another CTRO for $(\mathscr{L} \mathfrak{b}, \preccurlyeq)$}

There is another result establishing a CTRO for $(\mathfrak{L} \mathfrak{b}, \preccurlyeq)$, suggested by the compactness argument in $9^{\circ}$. It concerns not $\leqslant$ but yet another partial order on $\mathfrak{L b}$, which we write $\leqslant_{1}$. This time we assume that $(G, \leqslant, \preccurlyeq, \mathscr{U})$ is a locally compact TR group. In this case the set

$$
D_{1}=\{x \geqslant 0:[0, x] \text { is compact }\}
$$

is non-empty, and generates a subgroup $G_{1}=D_{1}-D_{1}$, for which $G_{1} \cap G^{+}=D_{1}$. For $f \in \mathfrak{Q b}(G)$ write

$$
f>{ }_{1} 0 \text { if and only if } f \geqslant 0 \text {, and } f(x)>0 \text { for every } x \in D_{1} \backslash\{0\} .
$$

This makes $\left(\mathfrak{L b}(G), \leqslant_{1}\right)$ a partially ordered vector space, and by almost the same arguments as were used in proving $3^{\circ}$ and $9^{\circ}$ we find that $\leqslant_{1}$, if non-trivial, is a TR $(2,2)$ determining order for $\preccurlyeq$ on $\mathfrak{Q b}(G)$, and $\left(\mathfrak{Q b}, \leqslant_{1}, \preccurlyeq\right)$ is a TRL group.

When $(G, \mathscr{U})$ is locally compact, $10^{\circ}$ is a particular case of this result.

\section{REFERENCES}

G. Birkhoff (1976), Lattice theory (Amer. Math. Soc., Providence, 3rd ed.).

F. F. Bonsall (1954), "Sublinear functionals and ideals in partially ordered vector spaces", Proc. London Math. Soc. (3) 4, 402-417.

N. Cameron and J. B. Miller (1975), "Topology and axioms of interpolation in partially ordered spaces", J. für die reine und angewandte Math. 278/279, 1-13.

A. Hayes (1962), "Additive functionals on groups", Proc. Camb. Phil. Soc. 58, 196-205.

P. Jaffard (1953), "Contribution à l'étude des groupes ordonnés", J. des Math. Pures et Appl. 32, 203-280.

R. J. Loy and J. B. Miller (1972), “Tight Riesz groups", J. Austral. Math. Soc. 13, 224-240.

J. B. Miller (1976), "Subdirect representation of tight Riesz groups by hybrid products", $J$. für die reine und angewandte Math. 283/284, 110-124. 
A. Peressini (1967), Ordered topological vector spaces (Harper and Row, New York).

R. H. Redfield (1976), "Non-secular, locally compact TRL groups" (Analysis Paper 18, Monash University, Melbourne).

A. Wirth (1975), "Locally compact tight Riesz groups", J. Austral. Math. Soc. 19, 247-251.

Monash University

Melbourne, Victoria 3168 